\title{
A Surveillance of Direct-Firing System for Pulverized-Coal Using Statistically Treated Signals from Intrusive Electrostatic Sensors
}

\author{
Boštjan Jurjevčič - Andrej Senegačnik - Igor Kuštrin* \\ University of Ljubljana, Faculty of Mechanical Engineering, Slovenia
}

Operational surveillance of all vital parts of thermal power plants is nowadays more important than any time before due to requirements for their extremely flexible operation resulting from intermittent behaviour of renewable energy sources. New methods for online measuring of pneumatic transport provide new possibilities for control and early fault detection of coal grinding and conveying system in direct-fired power plant boilers. Arrays of intrusive electrostatic sensors are an attractive option due to their inexpensive application and good spatial sensitivity required in large rectangular ducts of pulverized-coal systems. In this study, statistically treated electrostatic signals are used for detection of unexpected change in operating regime of coal grinding and conveying. Model-based and model-free autocorrelation reduction techniques are used to reduce the inherent autocorrelation of data. Forming batch-means of data, a model-free autocorrelation reduction technique is proposed in combination with an autoregressive-integrated-moving-average (ARIMA) method. Residuals between real and ARIMA-model fitted data are entered into exponentially-weighted-moving-average (EWMA) control chart for statistical surveillance of the process. The robust and cost-effective measuring method accompanied with a simple and intuitive control scheme proves to be effective for early fault detection of the pulverized-coal preparation system.

Keywords: ARIMA model, control chart, fan mill, fault detection, pneumatic transport, statistical modelling

Highlights

- Electrostatic method captures characteristics of gas-solid flow in the duct.

- Statistical process control uses electrostatic-sensors signals for monitoring of coal-grinding system.

- Successive autocorrelation reductions are employed: model-free and model-based.

- EWMA control chart of ARIMA-model residuals can detect unexpected coal-grinding regime changes.

\section{INTRODUCTION}

Thermal power plants are nowadays forced to operate in extremely flexible mode [1] to help maintaining balance between the electricity production and consumption [2] and [3]. Flexibility of pulverizedcoal direct-fired thermal power plant is limited by instability of combustion process at low boiler loads and sudden changes of coal composition. The need for measurement and control of every subsystem in the power plant to ensure stable operation and reduce emissions of harmful substances is necessary more than ever.

Characterization of pulverized coal pneumatic transport on its way from the mill to the furnace can provide important information and help improving the combustion [4] and [5]. Besides ensuring optimal pulverized-coal quantity and quality to burners, early detection of unexpected regime changes or faults is very welcome. Mill-overload condition is a very common and unwanted fault in pulverized-coal directfired steam boilers, which can cause large fluctuations in boiler's and consequently power plant's output [5] and [6]. Even a boiler trip can occur in the case of late or inappropriate intervention. Usually the danger of mill overloading is detected by observing the temperature of pulverized-coal-gas mixture exiting the mill [1] and [5]. Low temperature or increased gradient of temperature decrease of pulverizedcoal-gas mixture are usually used as triggers for mill-overload condition alarms. Experience shows that this type of detection can often be misleading or late. Besides this, mill's differential pressure and measurements of mill-motor amperage, vibrations [6], [7] and energy balance of mill [8] are used for monitoring of grinding and conveying system. In fact, controlled variables mentioned above are only indirectly related to coal flow. Nowadays, several techniques can be applied for direct observation of mass flow, velocity, concentration of particles, etc. in pneumatic transport [9] and [10], which have a great potential to improve control of grinding and transport process [6]. However, in case of direct-fired boilers with pulverized coal all known methods have limited applicability.

An electrostatic measuring technique based on intrusive sensors tested numerically [11] and experimentally [4] and [12] accompanied with appropriate data acquisition system is a promising one. Direct contact of sensors and coal-particles enables quick and adequate characterisation of the two-phase flow. Various signal-processing methods in time and frequency domain need to be applied to measured signal time series generated by electrostatic 
sensors, since raw data itself do not explicitly reflect flow characteristics. Mean or root-mean-square values of the signal with an appropriate calibration are typically employed.

Statistical analysis and modelling of time series can be applied for early detection of unexpected fluctuations of pneumatic transport. Various techniques can be applied for modelling of fluctuating time series [13]. For non-deterministic, stochastic time series [14], linear modelling based on statistical approach can be more appropriate than non-linear. Control charts are able to distinguish between common and special causes in numerous industrial applications [15], [16] and are widely used in control systems of thermal power plants [17] and [18]. Traditional Shewhart's, cumulative-sum (CUSUM) and exponentially-weighted-movingaverage (EWMA) control charts are frequently used in European power industry [19], [20] and can have better performance than non-standard charts [21] and [16]. CUSUM control chart weighs all past measurements equally. In order to detect sudden transients, frequent resetting of monitoring is inevitable. On the EWMA control chart, data is exponentially weighted. As data accumulate, the importance of past data diminishes. EWMA is therefore convenient for detection of quick transients while ignoring slow transients in non-stop operating processes. Furthermore, EWMA chart is simple and intuitive to implement and interpret [22] and [23].

The following sections describe the theoretical background of autocorrelation reduction and the basic principle of EWMA control charts. Afterwards, directfiring system of a coal fired boiler is briefly described. An array of electrostatic sensors is implemented as a source of data for further processing. EWMA control chart of treated data was tested in the case of regime transitions of direct-firing system and finally employed for early detection of mill-overload situation.

\section{THEORETICAL BACKGROUND}

For statistical process control, the assumption of data being independent and identically distributed is essential [24] and [25]. In reality, this is practically never the case. When the normality assumption is violated to a slight or moderate degree, control charts will still work reasonably well [24]. In contrast, conventional control charts do not perform well if the observations exhibit even low levels of correlation over time. Specifically, they will give misleading results in the form of too many false alarms if the data are positively correlated [20] and [23]. Nowadays we are frequently dealing with autocorrelated data because modern equipment enables high frequency sampling. Less frequent sampling can reduce the autocorrelation, but it can cause majority of information being disregarded [23]. Methods for the reduction of autocorrelation are needed.

Monitoring of autocorrelated processes by calculating the means of small batches of data separated by skipped observations was proposed in [26]. Moreover, better performance can be achieved by calculating batch-means without skipping any observations [25].

Another option to treat autocorrelated data is the algorithmic statistical process control. This technique incorporates the monitoring of residuals i.e. differences between actual and fitted data using an appropriate statistical model [16] and [27]. Autoregressive-integrated-moving-average (ARIMA) models appear to be a reasonable choice due to their high flexibility and need for very few assumptions [28].

\subsection{Model-Free Autocorrelation Reduction}

Unweighted batch means control chart is a form of model-free approach. It was proposed by [25] to break successive sequential observations into batches with equal weights assigned to every point in the batch. From the original time series of voltages $U_{1}, U_{2}, \ldots$, $U_{n}$ unweighted mean $U_{j}$ of $j^{\text {th }}$ batch can be calculated as:

$$
U_{j}=\frac{1}{b} \sum_{i=1}^{b} U_{b(j-1)+i} \quad \text { for } j=1,2, \ldots, m,
$$

where $b$ is the number of samples per batch. In [25] it is recommended to choose such a batch size that yields the autocorrelation to 0.1 at lag 1 . If time series is highly autocorrelated, increasing the batch size cannot sufficiently reduce the autocorrelation in reasonable lag time.

\subsection{Model-Based Autocorrelation Reduction}

The proposed model-based approach uses the ARIMA method. It incorporates a linear, strictly statistical model and a subset of time series analysis, developed by Box et al. [29]. Integers $p, d$ and $q$ are referring to autoregressive (AR), integrated (I) and moving average (MA) parts of the model respectively. The ARIMA method offers a potential for treating the time series that cannot be adequately treated by using an AR or an MA methods alone. Many advantages of the 
ARIMA method were found and support the adequacy of ARIMA especially for short term forecasting of time series. The ARIMA model can be written as:

$$
\left(1-\sum_{k=1}^{p} \alpha_{k} L^{k}\right)(1-L)^{d} U_{t}=\left(1+\sum_{k=1}^{q} \beta_{k} L^{k}\right) \varepsilon_{t}+\tilde{\mu}
$$

for $t=1,2, \ldots, n$ with:

$$
\tilde{\mu}=\mu-\alpha_{1} \mu-\ldots-\alpha_{p} \mu
$$

where $U_{t}$ is a measured value at time $t, \mu$ is a mean of past measured values, $\alpha_{1}, \ldots, \alpha_{p}$ are real numbers, $\varepsilon_{t}$ is a random error at time $t, \alpha_{k}$ are autoregression coefficients, $\beta_{k}$ is a moving average coefficient, $(1-L)$ is a differentiator operator and $L$ is a back shift operator. Past data $U_{1}, U_{2}, U_{3}, \ldots, U_{t-1}$ are used to determine values of model parameters, Eqs. (2) and (3). Model is then used for calculation of surrogate (fitted) data $\hat{U}_{t}$. The residual $e_{t}$ is calculated as:

$$
e_{t}=U_{t}-\widehat{U}_{t} \text {. }
$$

The residuals $e_{t}$ are approximately normally and independently distributed with zero mean and variance being constant [24].

\subsection{EWMA Control Chart}

While other control charts treat rational subgroups of samples individually, the EWMA chart tracks the exponentially-weighted-moving-average of all prior sample means. EWMA weights samples in geometrically decreasing order so that the most recent samples are weighted most highly, whereas the most distant samples are almost uninfluential [24]. According to EWMA, a smoothed exponentially weighted time series of calculated variables $z_{t}$ is defined as:

$$
z_{t}=\lambda \cdot e_{t}+(1-\lambda) z_{t-1}, \quad \text { for } t=1,2, \ldots, n,
$$

where $e_{t}$ is a residual at time $t$, and $0<\lambda \leq 1$ is a smoothing constant. Starting value $z_{0}$, which is required for calculation of $z_{1}$, is usually set to the mean of preliminary data. Lower $\lambda$ provides higher degree of the smoothing. Upper control limit $U C L_{t}(+)$ and lower control limits $L C L_{t}(-)$ are calculated as:

$$
C L_{t}= \pm k \sigma_{e, t} \sqrt{\frac{\lambda}{2-\lambda}\left[1-(1-\lambda)^{2 t}\right]}
$$

for $t=1,2, \ldots, n$, where $k$ is a control limit factor and $\sigma_{e, t}$ is a standard deviation of measured time series $e_{1}, e_{2}, \ldots, e_{t}$. Because the expression in square brackets with increasing number of samples converges to 1 , $U C L_{t}$ and $L C L_{t}$ approach constant values.

EWMA control chart can be applied to the sequence of residuals that are not autocorrelated if a model is properly chosen [27]. Either Shewhart or EWMA control charts of measured data can be employed for detection of regime change. In order to cover all regimes and simultaneously detect transitions between different regimes, it is more convenient to apply residuals instead of measured data into the control chart. Consequently, the control limits do not change at every change of regime, which is the case in conditional monitoring. The use of ARIMA residuals as EWMA inputs additionally reduces the autocorrelation and covers all regimes of operation.

\section{DESCRIPTION OF MEASURING PRINCIPLE AND DATA PROCESSING}

The method was employed and validated on a lignite grinding system of a $345 \mathrm{MW}$ power plant with directfiring system. Each of the six fan/impact-type mills feeds pulverized lignite to the four burner nozzles. The grinding system is shown in Fig. 1. Operating parameters are adjusted by changing the feeder and the mill rotational speed (Table 1). The pulverizedlignite conveying duct is physically divided into four partitions.

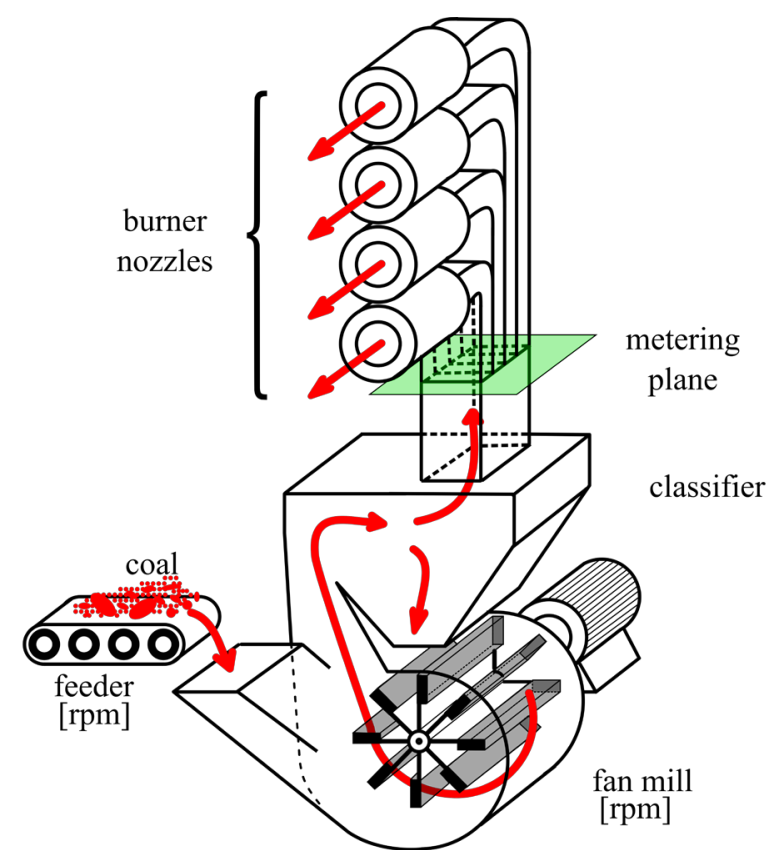

Fig. 1. Direct-firing thermal power plant's coal grinding and conveying system with burner nozzles 
Table 1. Operating regimes of fan mill and coal feeder

\begin{tabular}{|c|c|c|c|c|}
\hline \multirow{2}{*}{ Operating points } & M457 & M457 & M487 & M486 \\
\hline & F71 & F140 & $\mathrm{F} 70$ & F140 \\
\hline$n_{\text {mill }} \quad[\mathrm{rpm}]$ & 457 & 457 & 487 & 486 \\
\hline$n_{\text {feeder }}[\mathrm{rpm}]$ & 71 & 140 & 70 & 140 \\
\hline
\end{tabular}

Each partition leads to one of the four burner nozzles. The metering plane indicates the location where the electrostatic sensors are mounted.

The measuring system consists of eight rodtype electrostatic sensors: two sensors per one burner nozzle (Fig. 2). The system is described in detail in [4] and [5].

The signals of electrostatic sensors depend on the mass flow, the velocity, the electrical properties, the chemical composition of the particles and the dimensions of the sensors [4] and [30]. These relations have been used as a measuring principle for the velocity, the mass flow and the concentration of solid particles in many industrial applications. In this particular case, only the mass flow and the velocity of the particles can change rapidly enough to be qualified as a sudden transient. All other variables can be assumed being constant or at least very slowly changing. Each sensor is a source of its respective voltage time series. Therefore, the voltage time series sourced by each sensor can be written as:

$$
U_{u, t}=\sqrt{C \cdot v_{u, t}^{4} \cdot q_{m, u, t}} \quad t=1,2, \ldots, n,
$$

where $v$ and $q_{m}$ are the particles' velocity and mass flow and $C$ is the constant comprising all other less influential variables. Subscripts $u$ and $t$ refer to particular electrostatic sensor and time of sampling respectively. For further analysis, a time series of average voltage of all sensors $u_{1}$ to $u_{8}$ is used:

$$
U_{t}=\frac{1}{8} \sum_{u=1}^{8} U_{u, t}, \quad \text { for } t=1,2, \ldots, n .
$$

Time intervals between individual coal particle collisions to the electrostatic sensor are very short. For meaningful electrostatic-mass-flow or electrostaticconcentration measurements, which use the signals of the same sensors but they are not covered by this study, high sampling rate is required. Fluctuations of two-phase flow caused by more or less dense particle clusters are slower. Furthermore, combustion system response to certain occurrences and the operator ability to take actions are even slower. Therefore, high sample rates are needed to assess the characteristics of the pulverized-coal pneumatic transport. Frequent sampling causes neighbouring data to be highly autocorrelated which is undesirable for statistical analysis that requires the data to be independent [16] and [24]. In order to cover different time scales with minimal discard of information and at the same time reduce the autocorrelation, we propose the subsequent model-free and model-based autocorrelation reduction according to the following procedure:

- calculation of batch means of past measured voltages (model-free autocorrelation reduction),

- fitting of ARIMA model to the sequence of batch means and calculation of residuals i.e. differences between measured and ARIMA-model fitted voltages (model based autocorrelation reduction),

- EWMA control chart analysis of residuals.

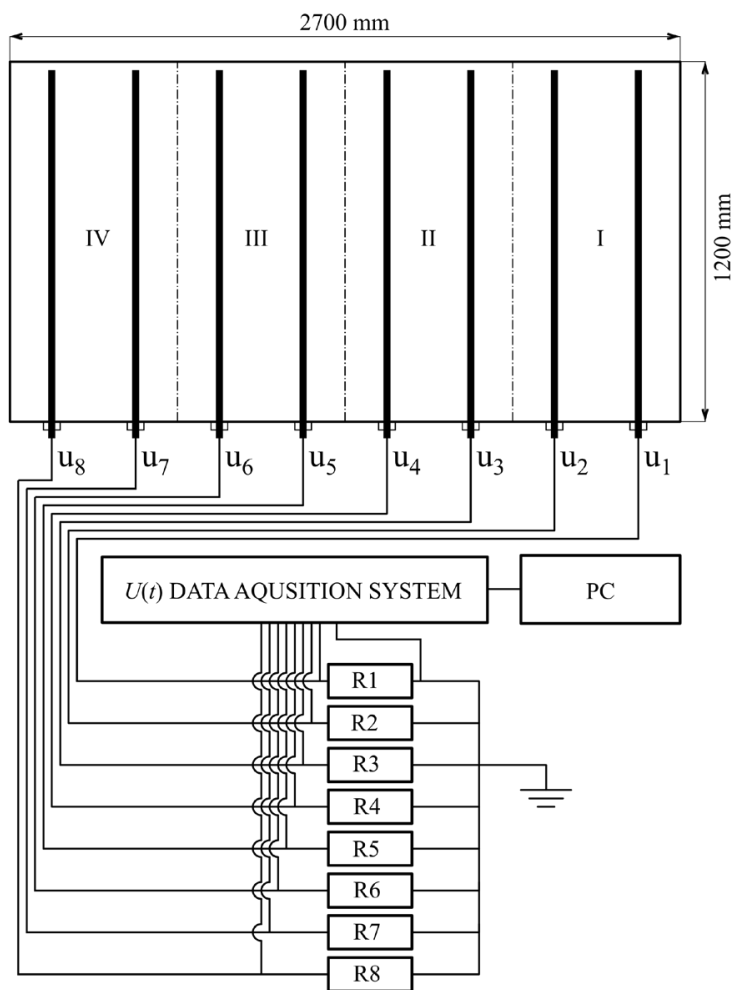

Fig. 2. Cross-section of the duct in the metering plane with four partitions (I - IV) and installation positions for rod-type sensors with measuring scheme

For the purpose of this analysis, two different types of transient situations are analysed:

- transitions between different operating regimes (Table 1),

- occurrence of mill overload condition.

The four operating regimes are described and commented in detail in [4]. In Table 1 feeder rotational speed is annotated by "F" and mill rotational speed by " $M$ ". Mill-overload condition is described and commented in detail in [5]. 
The total duration of the four operating regimes and accompanying transitions was 4 hours. Voltages were measured with a sample rate of $1000 \mathrm{~Hz}$. Two sets of data were archived:

- set of raw data: using temporary recorder,

- set of smoothed data: using power plant's installed data-acquisition and control system (DCS).

Smoothing was done by calculation the moving averages of five last means of batches each containing 1000 readings.

The total duration of the mill overload condition and its resolving was approximately 60 minutes. Only set of smoothed data was archived during this event using plant DCS.

The measurements acquired during the four regimes (Table 1) and the transitions between them were used for adjusting the parameters of batchmeans and ARIMA techniques. The measurements acquired during the mill-overload condition were used to verify the response of the proposed method to the development of a realistic and unwanted transient.

\section{RESULTS AND DISCUSSION}

\subsection{Time Series Analysis}

Fig. 3a shows 100-s interval of non-smoothed reduced voltage time series (Eq. 8) and smoothed time series acquired from electrostatic sensors in operating regime M486 F140. Coal-particles' collisions to the sensor and their moving through the sensing area are causing highly fluctuating stochastic signal. Smoothed time series is the moving average of five batch-means with batch size $b$ equal 1000 .

Power spectral density, as one of the most popular approach for fluctuating-time-series analysis, was applied to interval of the non-smoothed voltage time series as is shown in Fig. 3b. A broad peak in power occurs at relatively low frequencies (lower than $1 \mathrm{~Hz}$ ) and then power slightly decreases towards higher frequencies. At approximately $40 \mathrm{~Hz}$ the power starts to decrease more rapidly. Lower frequency fluctuations of the reduced voltage time series are caused by alternate motion of denser and diluter clusters of coal particles. The distinctive low frequencies for electrostatic measurements in twophase gas-solid flow are in compliance with other studies [11].

Autocorrelation function (Fig. 3c) evidently shows that the neighbouring data are highly correlated. Autocorrelation factor at lag 1 is 0.99 . The slowly decreasing autocorrelation function has a concentration of power spectral density in the lowest frequencies, which is in accordance with findings in [31].

\subsection{Autocorrelation Analysis and Autocorrelation Reduction}

High autocorrelation of the data is in stark contrast to data randomness that is an underlying assumption for statistic control of process. Autocorrelation needs to be reduced to an acceptable level. Approximately $95 \%$ of the sample autocorrelations should fall

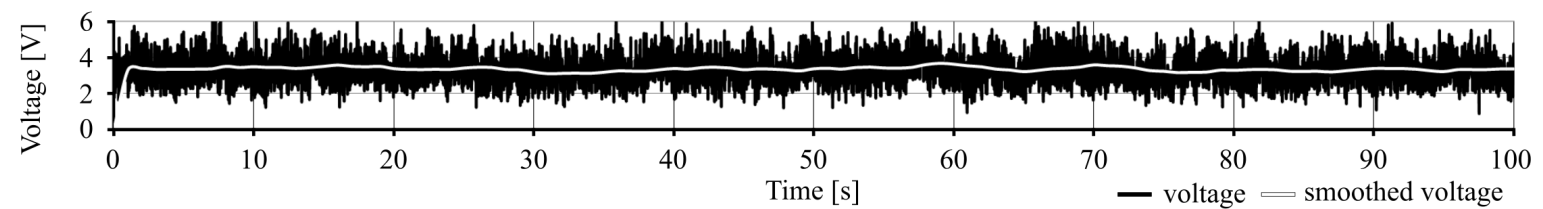

b)

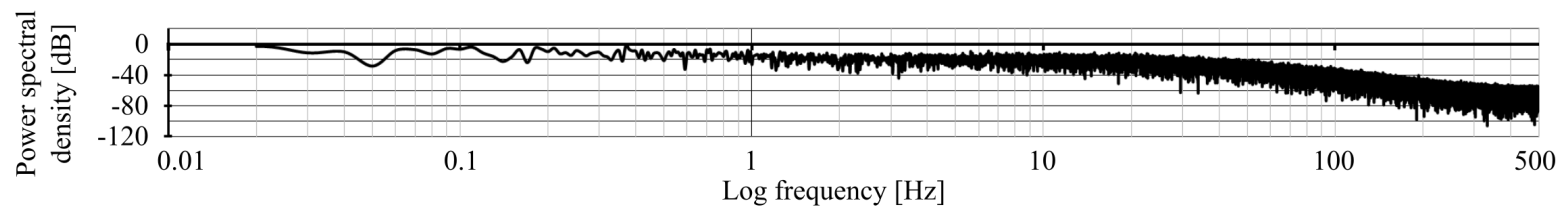

c)

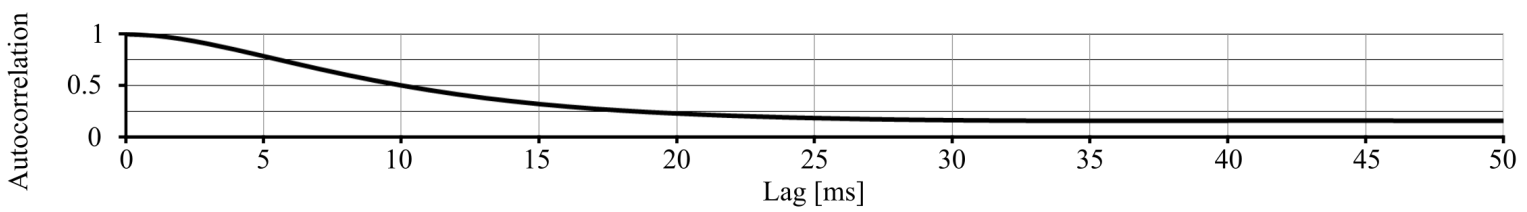

Fig. 3. a) Segment of non-smoothed and smoothed reduced voltage time series, b) power spectral density and c) autocorrelation plot of the reduced non-smoothed voltage for the operating regime M486 F140 
between the bounds $\pm 1.96 / \mathrm{n}^{1 / 2}$ since 1.96 is the 0.975 quantile of standard normal distribution [31].

Unweighted batch-means technique of autocorrelation reduction was applied. Autocorrelation functions for raw and smoothed reduced time series in the operating regime M486 F140 are shown on Fig. 4. The solid line refers to the time series of raw data before automatic smoothing (section 2: set of raw data).

Other lines refer to the reduced time series after the automatic smoothing. The long-dashed line shows autocorrelation function of automatically smoothed average time series before autocorrelation reduction (section 2, set of smoothed data, $b=1$ ).

The short-dashed line, the dotted-line and dash-dotted-line show autocorrelation functions for different degrees of autocorrelation reduction based on means of batches with $b=5, b=10$ and $b=30$ respectively. The smoothed time series has slightly lower autocorrelation factor at lag $=1$ than the rawdata time series. They both display high degree of autocorrelation and need autocorrelation reduction before being a suitable input to control chart. The autocorrelation reduction increases with increasing $b$, which is in accordance with [25]. It should be noted that for raw data lag $=1$ corresponds to $1 \mathrm{~ms}$, for batch size $b=1$ lag $=1$ corresponds to $1 \mathrm{~s}$, for batch size $b=5 \mathrm{lag}=1$ corresponds to $5 \mathrm{~s}$, etc.

Different operating regimes with their distinct velocities, mass flows and mean particles' sizes in pneumatic transport have significant influence on autocorrelation. Fig. 5 shows autocorrelation functions for smoothed reduced time series with $b=10$ for different operating regimes. Regimes at the higher feeder speed, i.e. higher mass flow, (M457 F140 and M486 F140) display higher degree of autocorrelation. Higher concentration of particles causes smoother flow, higher self-similarity in time and consequently higher autocorrelation. At the same feeder speed, regimes with the higher rotational speed of the mill display lower autocorrelation of data (M486 F140 vs M457 F140) due to more turbulent flow.

Even after application of batch means reduction technique, the autocorrelation is still too high for the assumption of randomness for all the observed operating regimes. The lowest autocorrelation factor at lag $=1$ and $b=10$ equals 0.29 and belongs to regime M487 F70.

Additional technique for reduction of autocorrelation is required. ARIMA models may be a suitable solution for highly autocorrelated data with predominantly low frequencies [31] and [29]. If model parameters are set correctly, the residuals of time series model can have negligible autocorrelation [24] and [25]. Table 2 comprises the results of different ARIMA model parameters $p, d$ and $q$. The ARIMA parameters were determined iteratively according to Akaike information criterion for finite samples (AICc). AICc is one of frequently used criterions [32]. The lowest AICc number indicates the best fitted model to data.

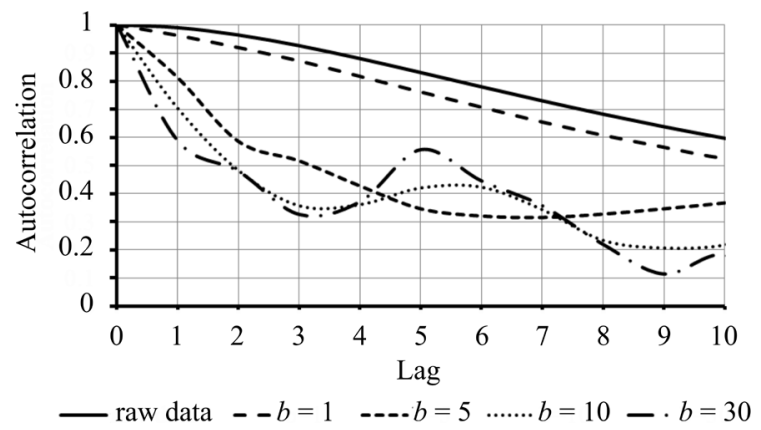

Fig. 4. Autocorrelation functions of raw and smoothed reduced time series with different batch sizes $b$ for operating regime M486 F140

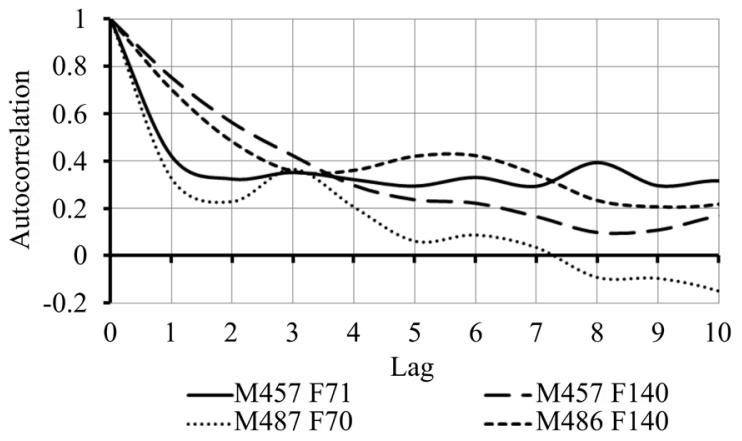

Fig. 5. Autocorrelation functions of batch-means of smoothed reduced time series, with $b=10$ for various operating regimes

Since different parameter values of ARIMA model were obtained for different operating regimes (Table 2) it would be more convenient to find a universal model that fits all regimes. For this purpose, average values of parameters of ARIMA models for all operating regimes for each batch size were calculated (third row for each batch size). The second row contains AICc values of best-fit ARIMA models for respective regimes. The fifth row contains AICc values of universal ARIMA model using nearest integers of average parameters of respective ARIMA models. Comparison of AICc values shows that the use of universal model is justifiable since it provides satisfactory results and simplifies the calculation procedure. 
Preceding batch-means autocorrelation reduction with larger $b$ reduces the values of ARIMA model parameters.

\subsection{Results of EWMA Control Chart Analysis}

The smoothed reduced time series were used for the analysis i.e. second type of data set (section 2). As mentioned above, the sets of data archived during transitions between operating regimes were used to choose the appropriate settings for autocorrelation reduction and control charts. Afterwards, the set of data archived during the mill-overload condition was used to verify the performance of the method during realistic unexpected fault in coal grinding and conveying system.

Table 2. ARIMA parameters and AICC values at the specific operating regimes and the surrogate model for various batch sizes

\begin{tabular}{|c|c|c|c|c|c|}
\hline & \multirow{2}{*}{ 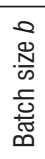 } & \multicolumn{4}{|c|}{ Operating regime } \\
\hline & & $\begin{array}{c}\text { M457 } \\
\text { F71 }\end{array}$ & $\begin{array}{r}\text { M457 } \\
\text { F140 }\end{array}$ & $\begin{array}{c}\text { M487 } \\
\text { F70 }\end{array}$ & $\begin{array}{c}\text { M486 } \\
\text { F140 }\end{array}$ \\
\hline$p, d, q$ & \multirow{5}{*}{1} & $3,1,1$ & $3,1,3$ & $4,1,3$ & $1,1,1$ \\
\hline $\operatorname{AICc}(p, d, q)$ & & -4927 & -5585 & -4709 & -4920 \\
\hline $\operatorname{avg} p, d, q$ & & \multicolumn{4}{|c|}{$2.75,1.00,2.00$} \\
\hline integer avg $p, d, q$ & & \multicolumn{4}{|c|}{$3,1,2$} \\
\hline AICc (integer avg $p, d, q$ ) & & -4933 & -5569 & -4660 & -4937 \\
\hline$p, d, q$ & \multirow{5}{*}{5} & $3,1,2$ & $1,0,2$ & $3,1,2$ & $1,1,2$ \\
\hline $\operatorname{AICc}(p, d, q)$ & & -602 & -892 & -697 & -716 \\
\hline $\operatorname{avg} p, d, q$ & & \multicolumn{4}{|c|}{$2.00,0.75,2.00$} \\
\hline integer avg $p, d, q$ & & \multicolumn{4}{|c|}{$2,1,2$} \\
\hline AICc (integer avg $p, d, q$ ) & & -603 & -883 & -694 & -714 \\
\hline$p, d, q$ & \multirow{5}{*}{10} & $1,1,1$ & $1,0,0$ & $0,1,1$ & $2,1,1$ \\
\hline $\operatorname{AICc}(p, d, q)$ & & -275 & -352 & -266 & -283 \\
\hline $\operatorname{avg} p, d, q$ & & \multicolumn{4}{|c|}{$1.00,0.75,0.75$} \\
\hline integer avg $p, d, q$ & & \multicolumn{4}{|c|}{$1,1,1$} \\
\hline AICc (integer avg $p, d, q$ ) & & -275 & -346 & -264 & -283 \\
\hline$p, d, q$ & \multirow{5}{*}{30} & $0,1,1$ & $1,0,0$ & $1,0,0$ & $0,1,1$ \\
\hline $\operatorname{AICc}(p, d, q)$ & & -120 & -102 & -117 & -94 \\
\hline $\operatorname{avg} p, d, q$ & & \multicolumn{4}{|c|}{$0.50,0.50,0.50$} \\
\hline integer avg $p, d, q$ & & \multicolumn{4}{|c|}{$1,1,1$} \\
\hline AICc (integer avg $p, d, q$ ) & & -118 & -96 & -110 & -92 \\
\hline
\end{tabular}

\subsubsection{EWMA Control Chart of Transitions Between Operating Regimes}

Besides the ARIMA residuals and EWMA of ARIMA residuals, the reduced voltage time series with $b=10$ is also plotted in Fig. 6 to illustrate the transitions between operating regimes. Smoothed residuals lying above the upper control limit (UCL) and below the lower control limit (LCL) are marked with red circles. Red circles only occur during transitions (A, B, C and D) and could be used as alarm for transients.

Table 3 shows effect of different input parameters to EWMA process control on the occurrence of alarms. Different batch sizes of model-free autocorrelation reduction and the corresponding universal ARIMA model from Table 2 were analysed along with different values of smoothing constant $\lambda$ for EWMA in the range 0.05 to 0.5 . The range of smoothing constant was chosen according to guidelines in [21]. Alarms appearing during stable operating regimes as well as in during transitions A, B, C and D were observed. Alarms occurring during the stable operating regime were denominated as false.

Table 3. EWMA control chart settings for alarms detection during operation in different regimes with control limit factor $k$ equal to 3

\begin{tabular}{|c|c|c|c|c|c|c|c|c|}
\hline & \multirow[t]{2}{*}{$b$} & \multirow[t]{2}{*}{$\lambda$} & \multicolumn{4}{|c|}{$\begin{array}{c}\text { Number of alarms in } \\
\text { transition }\end{array}$} & \multirow{2}{*}{$\begin{array}{l}\text { Number of } \\
\text { all alarms }\end{array}$} & \multirow{2}{*}{$\begin{array}{l}\text { Number of } \\
\text { false alarms }\end{array}$} \\
\hline & & & $A$ & $B$ & C & $D$ & & \\
\hline \multirow{6}{*}{ 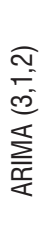 } & \multirow{6}{*}{1} & 0.05 & 6 & 0 & 0 & 2 & 11 & 3 \\
\hline & & 0.10 & 12 & 0 & 0 & 6 & 28 & 10 \\
\hline & & 0.20 & 15 & 3 & 0 & 11 & 62 & 36 \\
\hline & & 0.30 & 15 & 1 & 0 & 11 & 83 & 56 \\
\hline & & 0.40 & 15 & 1 & 0 & 10 & 86 & 60 \\
\hline & & 0.50 & 10 & 1 & 1 & 13 & 100 & 75 \\
\hline \multirow{6}{*}{ 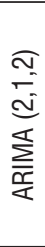 } & \multirow{6}{*}{5} & 0.05 & 9 & 4 & 0 & 4 & 17 & 0 \\
\hline & & 0.10 & 13 & 8 & 0 & 8 & 29 & 0 \\
\hline & & 0.20 & 22 & 6 & 1 & 9 & 38 & 0 \\
\hline & & 0.30 & 20 & 4 & 0 & 8 & 34 & 2 \\
\hline & & 0.40 & 18 & 3 & 0 & 8 & 30 & 1 \\
\hline & & 0.50 & 18 & 2 & 0 & 7 & 30 & 3 \\
\hline \multirow{6}{*}{$\begin{array}{l}\widetilde{E} \\
= \\
= \\
\sum_{\frac{1}{\alpha}}^{\frac{\alpha}{\alpha}}\end{array}$} & \multirow{6}{*}{10} & 0.05 & 2 & 7 & 2 & 0 & 11 & 0 \\
\hline & & 0.10 & 6 & 6 & 1 & 4 & 17 & 0 \\
\hline & & 0.20 & 7 & 5 & 2 & 5 & 19 & 0 \\
\hline & & 0.30 & 9 & 5 & 1 & 5 & 20 & 0 \\
\hline & & 0.40 & 10 & 4 & 1 & 5 & 20 & 0 \\
\hline & & 0.50 & 10 & 2 & 0 & 4 & 17 & 1 \\
\hline \multirow{6}{*}{ 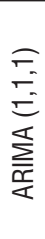 } & \multirow{6}{*}{30} & 0.05 & 0 & 0 & 0 & 0 & 0 & 0 \\
\hline & & 0.10 & 1 & 3 & 0 & 0 & 4 & 0 \\
\hline & & 0.20 & 1 & 3 & 1 & 1 & 6 & 0 \\
\hline & & 0.30 & 2 & 2 & 1 & 1 & 6 & 0 \\
\hline & & 0.40 & 2 & 2 & 1 & 2 & 7 & 0 \\
\hline & & 0.50 & 3 & 2 & 0 & 2 & 7 & 0 \\
\hline
\end{tabular}

The number of false alarms and total sum of all alarms decrease at higher batch size $b$. From this point of view, larger $b$ is a better choice. The most robust smoothing constant is 0.2 . Using this value, no falls alarms during stable regimes and at least one true alarm in transition $\mathrm{C}$ occur for all batch 
sizes. Average run length (ARL) is usually used for strictly mathematical evaluation of statistical process control scheme [23]. Lower smoothing constant $\lambda$ provides larger ARL values. When $\lambda$ approaches zero, ARL approaches infinity [22]. Our findings are in accordance with [23] and [24] where the range of smoothing constant was proposed to be $0.05<\lambda<$ 0.25 and is usually set to 0.2 .

\subsubsection{EWMA Control Chart of Mill-Overload Condition}

The chosen settings were tested using data archived during the unexpected mill-overload condition. This event is one of many archived by existing plant control system. All archived occurrences of milloverload condition are very similar and lead to the same conclusions.

Fig. 7 shows EWMA control chart with $\lambda=0.2$ for residuals of 10-s batch-means model-free and ARIMA $(1,1,1)$ model-based autocorrelation reductions carried out consecutively. Relative reduced voltage time series with batches of $10 \mathrm{~s}$ is shown on the lower section of the chart together with relative values of the classifier temperature, pulverized-coal velocity and the rotational speeds of feeder and mill rotor. Zero represents the lowest and unity represents the highest value of a respective variable. A lower control limit (LCL) of classifier temperature is set to $175{ }^{\circ} \mathrm{C}$. It is used for mill-overload detection with plant DCS.
More details about measuring these parameters can be found in [5].

Two periods are marked on the chart. Period A covers the coal accumulation in the mill because the mill was unable to properly grind and transport the coal to the burner nozzles. During this period voltage, pulverized coal velocity and classifier temperature decreased rapidly indicating obstructed grinding and transporting of the pulverized coal. Operator intervened i.e. reduced the feeder speed when the temperature crossed the temperature LCL.

During period B the feeder speed was slightly increased along with tree-step increase of mill rotational speed which enabled the mill to grind and transport the accumulated coal to the burners. The classifier temperature and pulverized coal velocity returned back to higher levels indicating that grinding and transporting is back to normal. The pulverizedcoal velocity was higher than in the period preceding the event because of lower coal mass flow. It must be noted that, despite lower coal mass flow, the voltage is higher than in the period preceding the event due to dominant effect of pulverized-coal velocity on the voltage (Eq. 1), [4]. EWMA control chart points out 12 alarms during period $\mathrm{A}$.

The first EWMA alarm occurred approximately 2.5 minutes prior to temperature alarm which is crucial for the operator. Early EWMA alarms allow less radical manual interventions and consequently alleviate stress situations for both personnel and power

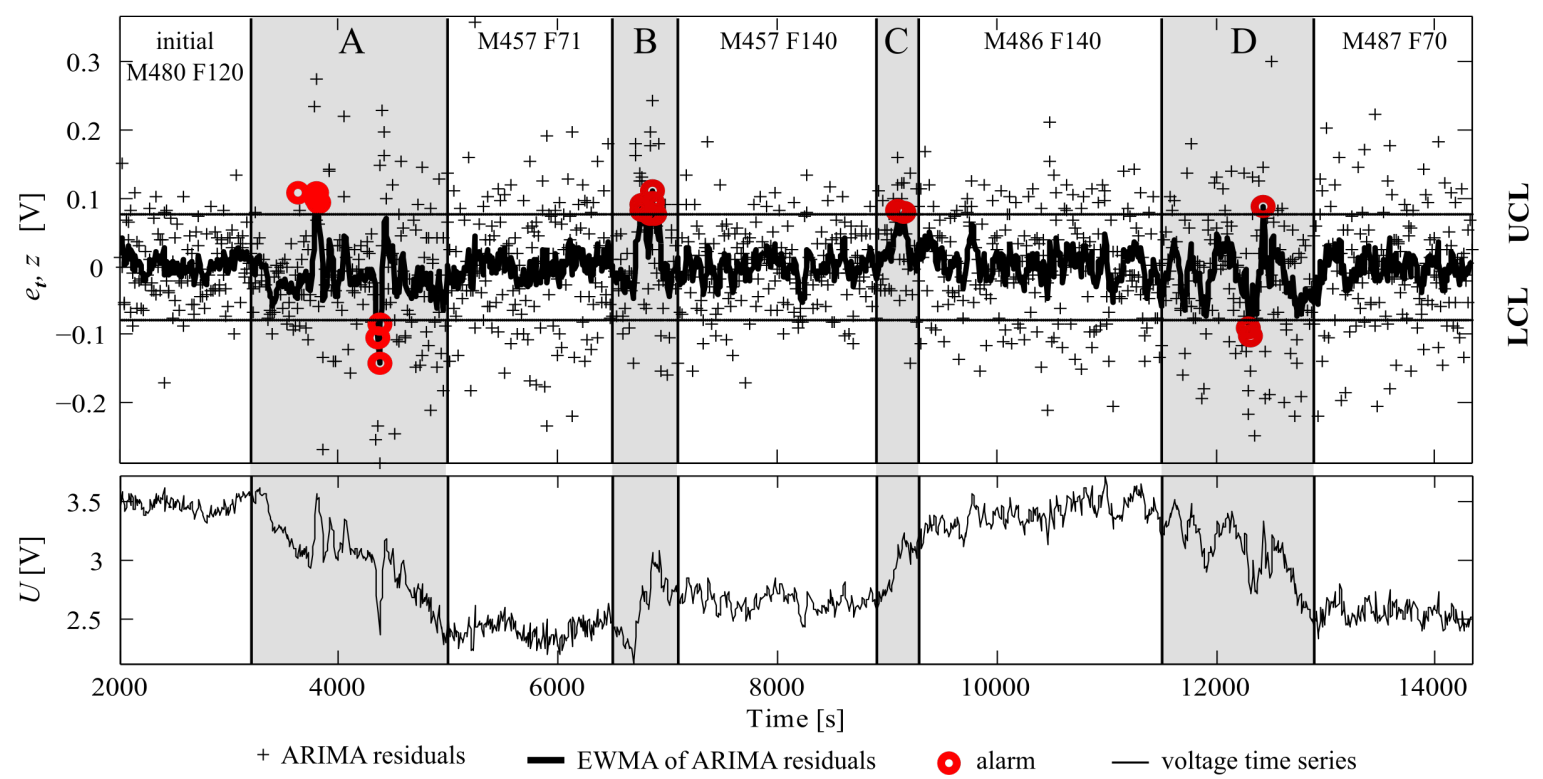

Fig. 6. EWMA control chart with smoothing constant $\lambda=0.2$ for residuals of 10 -s-batched ARIMA $(1,1,1)$ model with alarms in transitions A-D and reduced-smoothed voltage time series with $b=10$ through all the operating regimes 


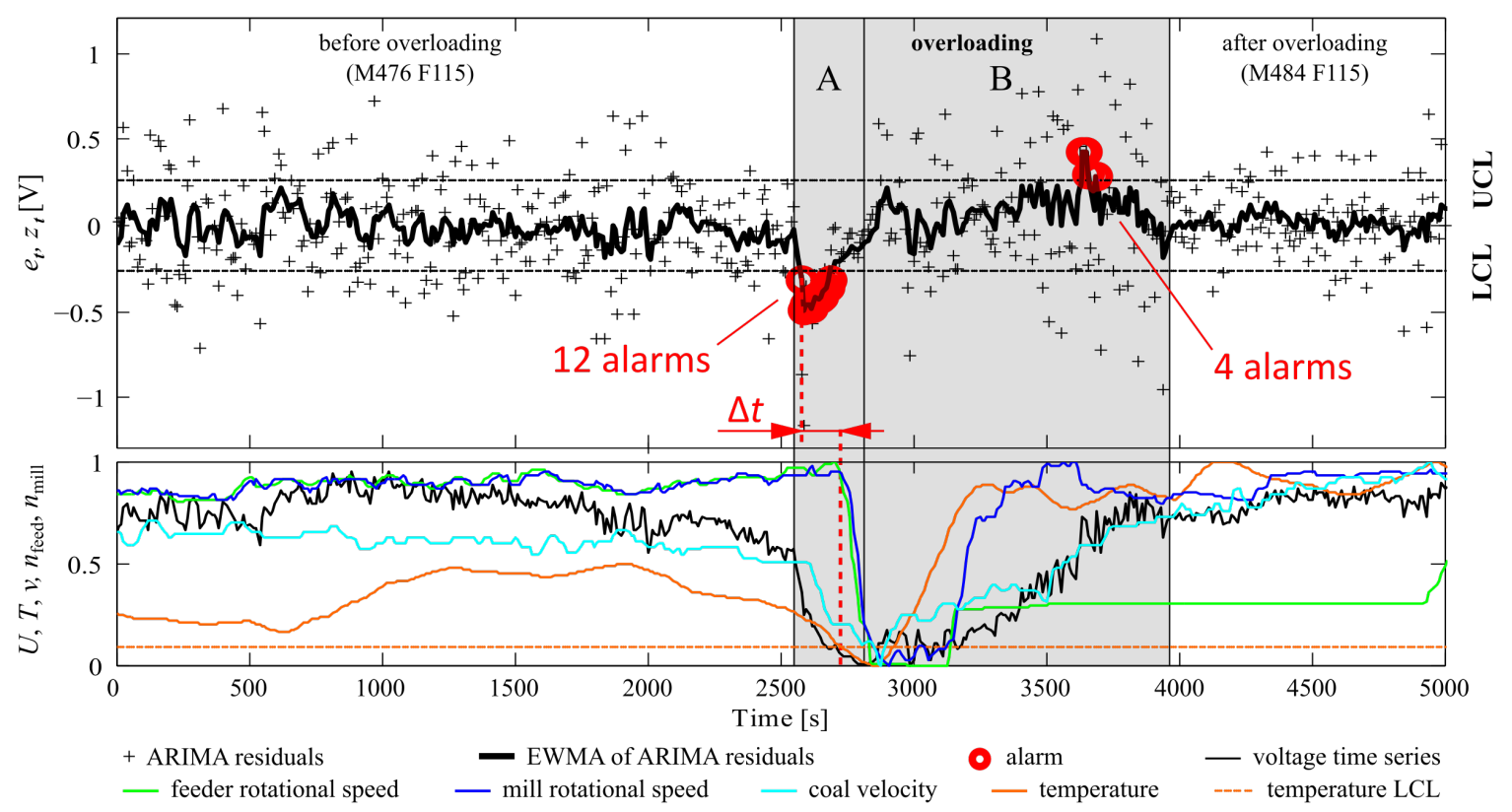

Fig. 7. EWMA control chart for overloaded mill and time series of voltage, feeder rotational speed, mill rotational speed, coal velocity, temperature in classifier and temperature lower control limit

plant system. Thus, sudden changes in combustion process can be prevented and consequently emission of harmful substances reduced. During period B four alarms occurred.These alarms are not considered false because they indicated actual sudden changes in operating regime caused by manual interventions.

\section{CONCLUSION}

The array of eight rod-type intrusive electrostatic sensors with accompanying instrumentation was employed in power plant's direct-fired boiler for measurements in pneumatic transport of pulverized coal. The archived voltage time series were statisticaly analysed. The most important conclusions regarding the use of electrostatic method for online measurements of pneumatic transport characteristics accompanied by statistical treating of measured signals are:

- The electrostatic measurement is robust and accurate enough to generate data which can be used for online statistical control of pneumatic transport.

- The inherent autocorrelation of acquired data needs to be reduced before statistic process control is applied. The combined model-free and ARIMA-model-based technique proved to be suitable for efficient autocorrelation reduction.

- EWMA control chart is simple to integrate into existing power plants' data acquisition and control systems for detection of potentially hazardous faults of coal-milling and conveying system. The batch size of data-batch-means variation was used to fine-tune and optimize the performance of EWMA control chart.

- Uncomplicated installation of electrostatic rod sensors with data acquisition system and simple control scheme of statistically treated data proved to be effective for early fault detection.

The presented technique proved to be applicable for early detection of mill-overload condition, which is only one of many potential faults of coal grinding and conveying systems. Other faults like unintentional increase of coal layer thickness caused by a foreign object, corrosion and abrasion induced feederbase-plate perforations or extreme slugging of hot recirculated-flue-gas ducts can also be detected by using this method. This particular method is able to trigger a reliable alarm few minutes earlier than other techniques that are traditionally used for detection of this kind of faults. This improvement is very important for thermal power plants continuously operating in load-following mode.

\section{REFERENCES}

[1] Mercangoez, M., Poland, J. (2011). Coal Mill Modeling for Monitoring and Control. IFAC Proceedings Volumes, vol. 44, no. 1, p. 13163-13166, D0I:10.3182/20110828-6IT-1002.01144. 
[2] Starkloff, R., Alobaid, F., Karner, K., Epple, B., Schmitz, M., Boehm, F. (2015). Development and validation of a dynamic simulation model for a large coal-fired power plant. Applied Thermal Engineering, vol. 91, p. 496-506, D0l:10.1016/j. applthermaleng.2015.08.015.

[3] Corn, M., Černe, G., Papič, I., Atanasijević-Kunc, M. (2014). Improved integration of renewable energy sources with the participation of active customers. Strojniški vestnik - Journal of Mechanical Engineering, vol. 60, no. 4, p. 274-282, DOl:10.5545/sv-jme.2013.1199.

[4] Jurjevčič, B., Senegačnik, A., Drobnič, B., Kuštrin, I. (2015). The characterization of pulverized-coal pneumatic transport using an array of intrusive. IEEE Transactions on Instrumentation and Measurement, vol. 64, no. 12, p. 34343443, D0I:10.1109/TIM.2015.2465731.

[5] Kustrin, I., Lenart, J. (2015). Electrostatic sensors on a lignite - fired boiler for continuously monitoring the distribution and velocity of pulverized coal. VGB Powertech, no. 7, p. 33-37.

[6] Agrawal, V., Panigrahi, B.K., Subbarao, P.M.V. (2014). Review of control and fault diagnosis methods applied to coal mills. Journal of Process Control, vol. 32, p. 138-153, D0l:10.1016/j. jprocont.2015.04.006.

[7] Doukovska, L., Vassileva, S. (2013). Knowledge-based mill fan system technical condition prognosis. WSEAS Transactions on Systems, vol. 12, no. 8, p. 398-408.

[8] Odgaard, P.F., Mataji, B. (2007). Fault detection in coal mills used in power plants. IFAC Proceedings Volumes, vol. 39, no. 7, p. 177-182, D0l:10.3182/20060625-4-CA-2906.00036.

[9] Yan, Y. (2001). Guide to the flow measurement of particulate solids in pipelines, International Journal of Storing, Handling and Processing Powder, vol. 13, no. 4, p. 343-352.

[10] Blondeau, J., Kock, R., Mertens, J., Eley, A.J., Holub, L. (2016). Online monitoring of coal particle size and flow distribution in coal-fired power plants: Dynamic effects of a varying mill classifier speed. Applied Thermal Engineering, vol. 98, p. 449454, DOI:10.1016/j.applthermaleng.2015.12.113.

[11] Zhe, K., Xiao-lei, W., Shu-jiang, Z. (2013). Study on the spatial filtering and sensitivity characteristic of inserted electrostatic sensors for the measurement of gas-solid two-phase flow parameters. Flow Measurement and Instrumentation, vol. 30, np. 26-33, Dol:10.1016/j.flowmeasinst.2012.10.001.

[12] Shao, J., Krabicka, J., Yan, Y. (2010). Velocity Measurement of pneumatically conveyed particles using intrusive electrostatic sensors. IEEE Transactions on Instrumentation and Measurement, vol. 59, no. 5, p. 1477-1484, D0l:10.1109/ TIM.2010.2040960.

[13] Potočnik, P., Strmčnik, E., Govekar, E. (2015). Linear and neural network-based models for short-term heat load forecasting. Strojniški vestnik - Journal of Mechanical Engineering, vol. 61, no. 9, p. 543-550, D0l:10.5545/sv-jme.2015.2548.

[14] Jurjevčič, B. (2017). The nonlinear analysis of time series from intrusive-electrostatic sensors for gas-solid-flow characterization, yearbook, Department of Energy Engineering, Faculty of Mechanical Engineering, Ljubljana.

[15] Zhao, Y., Wang, S., Xiao, F. (2013). A statistical fault detection and diagnosis method for centrifugal chillers based on exponentially-weighted moving average control charts and support vector regression. Applied Thermal
Engineering, vol. 51, no. 1-2, p. 560-572, D0l:10.1016/j. applthermaleng.2012.09.030.

[16] Kandananond, K. (2014). Guidelines for applying statistical quality control method to monitor autocorrelated processes. Procedia Engineering, vol. 69, p. 1449-1458, D0l:10.1016/j. proeng.2014.03.141.

[17] Graham, D.P., Salway, G. (2015). Electricity Supply Industry IED Compliance Protocol for Utility Boilers and Gas Turbines, E.ON Technologies, Ratcliffe.

[18] Kisić, E., Petrović, V., Jakovljević, M., Đjurović, Ž. (2013). Fault detection in electric power systems based on control charts. Serbian Journal of Electrical Engineering, vol. 10, no. 1, p. 7390, D0I:10.2298/SJEE1301073K.

[19] Graham, D. (2010). European Power Industry Experience of EN14181, VGB powertech, vol. 90, no. 1-2, pp. 83-94.

[20] Deeskow, P., Steinmetz, U., Hay, M. (2008). Datamining und statistische Prozesskontrolle zur zustandsorientierten Instandhaltung. VGB powertech, vol. 88, no. 10, p. 84-87.

[21] Apley, D.W., Cheol Lee, H. (2003). Design of exponentially weighted moving average control charts for autocorrelated processes with model uncertainty. Technometrics, vol. 45, no. 3, p. 187-198, D0l:10.1198/004017003000000014.

[22] Lazariv, T., Okhrin, Y., Schmid, W. (2015). Behavior of EWMA type control charts for small smoothing parameters. Computational Statistics \& Data Analysis, vol. 89, p. 115-125, D0l:10.1016/j.csda.2015.03.010.

[23] Lucas, J.M., Saccucci, M.S. (1990). Exponentially Moving weighted schemes: control average and enhancements properties. Technometrics, vol. 32, no. 1, p. 1-12, D0I:10.1080 /00401706.1990.10484583.

[24] Montgomery, D. (2009). Introduction to Statistical Quality Control, $6^{\text {th }}$ ed. John Wiley and Sons Ltd., Chichester.

[25] Runger, G.C., Willemain, T.R. (1996). Batch-means control charts for autocorrelated data. IIE Transactions, vol. 28, no. 6, p. 483-487, D0I:10.1080/07408179608966295.

[26] Alwan, L.C., Radson, D. (1992). Time-series investigation of subsample mean charts. IIE Transactions, vol. 24, no. 5, p. 6680, DOI:10.1080/07408179208964246.

[27] Russo, S.L., Camargo, M.E., Fabris, J.P. (2012). Applications of Control Charts Arima for Autocorrelated Data. Practical Concepts of Quality Control, Nezhad, M.S.F. (ed.), InTech, p. 31-53, D0I:10.5772/50990.

[28] Ho, S.L., Xie, M. (1998). The use of ARIMA models for reliability forecasting and analysis. Computers \& Industrial Engineering, vol. 35, no. 1-2, p. 213-216, D0l:10.1016/S03608352(98)00066-7.

[29] Box, G.E.P., Jenkins, G., Reinsel, G.C. (2008). Time Series Analysis: Forecasting and Control, $4^{\text {th }}$ ed., Wiley, Hoboken, DOl:10.1002/9781118619193.

[30] Zhang, J. (2012). Air-solids flow measurement using electrostatic techniques, Electrostatics, Canbolat, H. (ed.), InTech, Rijeka, p. 61-80, D0I:10.5772/35937.

[31] Brockwell, P.J., Davis, R.A. (2002). Introduction to Time Series and Forecasting, $2^{\text {nd }}$ ed., Springer, New York, D0l:10.1007/ b97391.

[32] Burnham, K.P., Anderson, D.R. (2013). Model Selection and Multimodel Inference: A Practical Information-Theoretic Approach, $2^{\text {nd }}$ ed., Springer, New York, D0l:10.1007/b97636. 